DESY 98-170

hep-ph/9811519

November 1998

\title{
Less Singular Terms and Small $x$ Evolution in a Soluble Model ${ }^{\mathbb{}}$
}

\author{
J. Blümlein and W. L. van Neervenf \\ DESY-Zeuthen \\ Platanenallee 6, D-15735 Zeuthen, Germany
}

November 1, 2018

\begin{abstract}
We calculate the effect of the less singular terms at small $x$ on the evolution of the coefficient function in $\phi^{3}$ theory in six dimensions, which result from a complete solution of the ladder equation. Scale-invariant next-to-leading order contributions are also studied. We show that the small- $x$ approximation does not deliver the dominant contributions.
\end{abstract}

PACS numbers : 12.38.Cy

\footnotetext{
${ }^{1}$ Work supported in part by EU contract FMRX-CT98-0194(DG 12 - MIHT)

${ }^{2}$ On leave of absence from Instituut-Lorentz, University of Leiden, P.O. Box 9506 , 2300 RA Leiden, The Netherlands
} 
The resummation of the terms of the type $\left(\ln ^{i} x\right) / x$ [1, 2], occurring in the flavor singlet evolution kernels of the deep inelastic scattering structure functions at small values of $x=Q^{2} /(2 p . q)$, leads to large effects [3, 田. If one includes the next-to-leading order small $x$ terms, the effects are so strong that the resummed gluon splitting function becomes already negative at $x$-values as large as $10^{-2}$ for $Q^{2} \simeq 20(\mathrm{GeV} / c)^{2}$ [4]. Moreover the corrections to the coefficient $\omega$ describing the large energy $(\sqrt{s})$ behavior of the inclusive cross sections, given by $s^{\omega}$, may lead to negative values of $\omega$ [5]. Already in [6] the question was raised to which extent the leading logarithms dominate the perturbation series of the kernels or that less-singular terms are also important. For the case of the splitting functions and coefficient functions, out of which the kernels are constituted, this is shown in fixed-order perturbation theory in [4, 6]. A relation between the most singular and less singular terms at $x=0$ is for instance given by the conservation laws, as fermion number [7] and energy-momentum conservation [4, 8]. As outlined in Refs. [4, 9] the less singular contributions may cure the large effects in the evolution kernels caused by the resummation of the leading logarithms mentioned above.

In QCD the resummation of the most singular term in the leading order kernel is achieved by the leading order BFKL-equation [1]. Unfortunately until now one is not able to resum the full kernel so that the subleading contributions cannot be included in the resummation. Therefore one has no real check on the validity of the small $x$ approximation for the high energy behavior of the cross section or the small $x$ behavior of the structure functions. However in the case of massless $\phi_{6}^{3}$-theory the resummation of the exact leading order kernel is known [10] so that the validity of the small- $x$ approximation can be tested here. This will be our goal in this paper where we obtain information about the rôle of the less-singular terms in the kernel which are neglected in the BFKL approach. In particular we study the difference between the exact and approximate resummation for the splitting functions and the coefficient functions where we also discuss some contributions from the next-to-leading order kernel.

The Bethe-Salpeter equation for the forward scattering amplitude $T(p, q)$ in massless $\phi^{3}$ theory is given by

$$
T(q, p)=\frac{2^{2-D}}{\pi^{D / 2} \Gamma[(D-2) / 2]} \frac{\lambda^{2}}{(q-p)^{2}}+\frac{1}{(2 \pi)^{D}} \int d^{D} k \frac{\lambda^{2} T(k, p)}{(q-k)^{2}\left[k^{2}\right]^{2}} .
$$

Here, $\lambda$ denotes the coupling constant and $D$ is the dimension of space-time. Furthermore the leading order kernel is given by the propagator $1 /(q-p)^{2}$. For $D=6$ the quantity $q . p T(q, p)$ becomes scale invariant. The equation above is solved by expanding all functions in terms of Gegenbauer polynomials which is the analogue of the partial wave expansion in three dimensions in terms of Legendre functions. The leading $q^{2}$-behavior of the Nth partial wave $T_{N}(q, p)$ is given by $[10$

$$
\begin{aligned}
& p . q T_{N}(q, p) \sim p . q T_{N}^{(0)}(p, q) \hat{T}_{N}\left(\frac{q^{2}}{p^{2}}\right) \quad p^{2}<0, \quad q^{2}<0, \quad \text { with } \\
& p . q T_{N}^{(0)}(p, q)=\left(\frac{q^{2}}{p^{2}}\right)^{-(N+1) / 2}, \quad \hat{T}_{N}\left(\frac{q^{2}}{p^{2}}\right)=\left(\frac{q^{2}}{p^{2}}\right)^{-\gamma_{L}\left(N, a_{s}\right) / 2}
\end{aligned}
$$

with

$$
\gamma_{L}\left(N, a_{s}\right)=\sqrt{(N+2)^{2}+1-2 \sqrt{(N+2)^{2}+4 a_{s}}}-(N+1)=\sum_{k=0}^{\infty} a_{s}^{k} \gamma_{L}^{(k)}(N)
$$


and $a_{s}=\lambda^{2} /(4 \pi)^{3}=\alpha_{s} /(4 \pi)$. The quantity above is nothing but than the ladder approximation to the anomalous dimension of the composite operator of spin $N$ given by $\phi \partial_{\mu_{1}} \cdots \partial_{\mu_{N}} \phi$ in $\phi_{6}^{3}$ theory. This we have verified by a fixed-order calculation up to three-loop order. Because of scale invariance the quantity $\hat{T}_{N}(q, p)$ satisfies the scaling equation

$$
\left[p \frac{\partial}{\partial p}-\gamma_{L}\left(N, a_{s}\right)\right] \hat{T}_{N}\left(\frac{q^{2}}{p^{2}}\right)=0
$$

which equals to the renormalization group equation in the conformal limit. The splitting functions and the coefficient functions, both depending on the variable $x$, are related to the quantities above via a Mellin transformation. The splitting function is given by

$$
\gamma\left(N, a_{s}\right)=-\int_{0}^{1} d x x^{N-1} P\left(x, a_{s}\right)
$$

and the $Q^{2}$-dependence of the coefficient function $C$ is determined by the anomalous dimension via the relation

$$
\left(\frac{Q^{2}}{Q_{0}^{2}}\right)^{-\gamma\left(N, a_{s}\right) / 2}=\int_{0}^{1} d x x^{N-1} C\left(x, Q^{2}, \alpha_{s}\right), \quad q^{2}=-Q^{2},
$$

which satisfies Eq. (位).

Since the full leading order kernel has been resummed by Eq. (11) the lowest order coefficient of the ladder approximation coincides, up to the constant $1 / 6$ due to energy-momentum conservation, with the exact one given by $\gamma_{S S}^{(0)}(N)$,

$$
\gamma_{S S}^{(0)}(N)=-\frac{2}{(N+1)(N+2)}+\frac{1}{6} .
$$

However, no resummation exists for the next-to-leading kernel and we only know the exact order $\alpha_{s}^{2}$-contribution to the anomalous dimension [11] which are given in the $\overline{\mathrm{MS}}$-scheme by

$$
\gamma_{S S}^{(1)}(N)=\gamma_{S S}^{(1, a)}(N)+\frac{1}{2}\left[1+(-1)^{N}\right] \gamma_{S S}^{(1, b)}(N)
$$

with

$$
\begin{aligned}
& \gamma_{S S}^{(1, a)}(N)=\frac{5}{3} \frac{S_{1}(N)}{(N+1)(N+2)}-\frac{1}{6} \frac{22+111 N+211 N^{2}+138 N^{3}+28 N^{4}}{(N+1)^{3}(N+2)^{3}}+\frac{13}{216}, \\
& \gamma_{S S}^{(1, b)}(N)=-\frac{2}{(N+1)^{2}(N+2)^{2}} .
\end{aligned}
$$

Here $\gamma_{S S}^{(1, a)}(N)$ is due to the ladder-, vertex-, and self energy contributions. The contribution $\gamma_{S S}^{(1, b)}(N)$ is obtained from the crossed-ladder graph which like the planar ladder graphs, leading to $\gamma_{L}\left(N, a_{s}\right)$, satisfies the scaling equation (4). From the Mellin transforms in Eqs. (5.6) and the anomalous dimensions given above one infers that the most singular behavior of the splitting functions and the coefficient functions at $x=0$ is determined in $\phi_{6}^{3}$-theory by the leading pole terms of the type $O\left[\left(a_{s} /(N+1)\right)^{k}\right]$. Notice that the latter are shifted by two units with respect to those given in QCD of $O\left[\left(a_{s} /(N-1)\right)^{k}\right]$. Taking the limit $N \rightarrow-1$ in $\gamma_{S S}^{(i)}\left(N, a_{s}\right)(i=0,1)$ one finds that the most singular contributions $\propto\left[a_{s} /(N+1)\right]^{k}$ are all contained in the ladder approximation $\gamma_{L}\left(N, a_{s}\right)$. 


\begin{tabular}{||r||r|r|r|r|r||}
\hline \hline$k$ & \multicolumn{1}{|c|}{$c_{0, k}$} & \multicolumn{1}{c|}{$c_{1, k}$} & \multicolumn{1}{c|}{$c_{2, k}$} & \multicolumn{1}{c|}{$c_{3, k}$} & \multicolumn{1}{c|}{$c_{4, k}$} \\
\hline \hline 1 & -2 & 2 & -2 & 2 & -2 \\
2 & -2 & 4 & -4 & 2 & 2 \\
3 & -4 & 12 & -20 & 24 & -24 \\
4 & -10 & 40 & -88 & 140 & -180 \\
5 & -28 & 140 & -380 & 740 & -1156 \\
6 & -84 & 504 & -1624 & 3724 & -6804 \\
7 & -264 & 1848 & -6888 & 18144 & -37856 \\
8 & -858 & 6864 & -29040 & 86328 & -202248 \\
9 & -2860 & 25740 & -121863 & 403260 & -1047420 \\
10 & -9724 & 97240 & -509080 & 1856140 & -5291572 \\
\hline \hline
\end{tabular}

table 1: The first expansion coefficients of Eq. (12).

In analogy to the leading order BFKL-equation [1] one may determine now the all-order small- $x$ limit of the coefficient function via Eq. (6). For this purpose we expand $\gamma_{L}\left(N, a_{s}\right)$ into an infinite series in $a_{s}$ and collect all terms of $O\left[\left(a_{s} /(N+1)\right)^{k}\right]$, which can be represented in a closed form again [12],

$$
\gamma_{L, N \rightarrow-1}\left(N, a_{s}\right)=\sum_{k=1}^{\infty} c_{0, k} \frac{a_{s}^{k}}{(N+1)^{2 k-1}} \equiv(N+1)\left[\sqrt{1-\frac{4 a_{s}}{(N+1)^{2}}}-1\right] .
$$

Performing the same series expansion on the exact ladder approximation in Eq. (3) one can study the size of all terms which are less singular at $N=1$ than those contained in the approximate expression above. The series expansion of Eq. (3) is given by

$$
\gamma_{L}\left(N, a_{s}\right)=\sum_{k=1}^{\infty} \sum_{l=0}^{\infty} c_{l, k} \frac{a_{s}^{k}}{(N+1)^{2 k-1+l}} .
$$

A comparison between the residues of the leading pole terms given by $c_{0, k}$ and the residues corresponding to the subleading contributions $c_{l, k}(l \geq 1)$ is made in table 1. From the table we infer that the residues of the less singular pole terms show alternating signs and are of larger modulus. Therefore the leading series in Eq. (11) receives large corrections and is not dominant. A similar behavior was observed for the anomalous dimensions and coefficient functions in fixedorder perturbation theory in QCD before [4, 6].

To illustrate the importance of the subleading pole terms we have computed various splitting functions from the anomalous dimensions listed above by inverse Mellin transformation (see Eq. (55) ) for $10^{-10}<x<1$ in figure 1 for $\alpha_{s}=0.2$. All curves are normalized to the leading order splitting function for $x<1, a_{s} P_{L}^{(0)}(x)=2 a_{s} x(1-x)$. The splitting function in next-toleading order $a_{s} P_{S S}^{(0)}(x)+a_{s}^{2} P_{S S}^{(1)}(x)$ (Eqs. (7), 8$)$ ) shows a relative rise towards small $x$ compared to the leading order result Eq. (8). The resummation of all ladder graphs, $P_{L}$ Eq.(3)), reveals an appreciable contribution to the exact order $\alpha_{s}^{2}$ corrected splitting function. However this effect is not due to the leading pole terms $O\left[\left(a_{s} /(N+1)\right)^{k}\right]$ or most singular $x$-contributions $O\left(x \ln ^{k-1} x\right)$. This is shown by the plot of the small- $x$ approximation $P_{L, x \rightarrow 0}$ which is derived from Eq. (11). Here we see a large discrepancy between the exact ladder resummation (Eq. (3)) and its small- $x$ or leading pole approximation (Eq. (11)). This becomes even larger when $x$ gets smaller, where the approximation above is supposed to hold. 
The features shown for the splitting functions also hold for the coefficient functions. This is revealed in figure 2 where we have plotted the ratio $C_{L, x \rightarrow 0}\left(x, Q^{2}, \alpha_{s}\right) / C_{L}\left(x, Q^{2}, \alpha_{s}\right)$. Here $C_{L}$ and $C_{L, x \rightarrow 0}$ are determined via Eq. (6) by Eq. (3) and Eq. (11), respectively. The ratio illustrates the behavior of the structure function idealized by a $\delta(1-x)$-like parton distribution in the conformal limit. Like in the case of the splitting functions the discrepancy between the exact and small- $x$ resummation becomes larger when $x$ gets smaller. This reveals again that the leading pole terms or small- $x$ terms do not constitute the bulk of the resummed laddersolution. The next-to-leading order solution of the Bethe-Salpeter equation in (1) also involves the resummation of the crossed ladder graph, of which the order $\alpha_{s}^{2}$ contribution to the anomalous dimension is given in Eq. (10). Addition of the latter contribution to $\gamma_{L}$ in Eq. (3) leads to a positive contribution to $C_{L}$ so that the ratio decreases. The latter also happens when $Q^{2}$ in Eq. (6) or the fixed coupling constant $\alpha_{s}$ gets smaller.

In summary it was shown that in $\phi_{6}^{3}$-theory the less singular contributions in the anomalous dimension, which are obtained by the complete ladder solution of the Bethe-Salpeter equation lead to sizeable corrections to the resummation of the small- $x$ terms in Eq. (11). These contributions lead to a smaller rise of the coefficient function at small $x$. One might wish to obtain a similar complete conformal solution also in QCD. As signaled by the behavior of the fixed-order results such a resummation could lead to a more stable solution at small $x$ than shown by the currently available resummations [1, 2].

Acknowledgment. For discussions we would like to thank V. Ravindran and A. Vogt.

\section{References}

[1] E.A. Kuraev, L.N. Lipatov, and V.S. Fadin, Sov. Phys. JETP 45 (1977) 199; Y. Balitskii and L.N. Lipatov, Sov. J. Nucl. Phys. 28 (1978) 822.

[2] S. Catani and F. Hautmann, Nucl. Phys. B427 (1994) 475; V.S. Fadin and L.N. Lipatov, Phys. Lett. B429 (1998) 127; M. Ciafaloni and G. Camici, Phys. Lett. B430 (1998) 349.

[3] J. Blümlein, S. Riemersma, and A. Vogt, hep-ph/9608470, Nucl. Phys. B (Proc. Suppl.), 51C (1996) 30; J. Blümlein and A. Vogt, Phys. Rev. D57 (1998) R1.

[4] J. Blümlein and A. Vogt, Phys. Rev. D58 (1998) 014020.

[5] J. Blümlein, V. Ravindran, and W.L. van Neerven, Phys. Rev. D58 (1998) 091502.

[6] J. Blümlein, talk, Durham HERA Workshop, March 1993 and J. Phys. G19 (1993) 1695; W.L. van Neerven, talk, DESY Theory Workshop, September 1993.

[7] J. Blümlein and A. Vogt, Phys. Lett. B370 (1996) 149; Acta Phys. Pol. B27 (1996) 1309.

[8] R.K. Ellis, F. Hautmann and B. Webber, Phys. Lett. B348 (1995) 582.

[9] J. Blümlein, V. Ravindran, W.L. van Neerven, and A. Vogt hep-ph/9806368.

[10] C. Lovelace, Phys. Lett. 55B (1975) 187, Nucl. Phys. B95 (1975) 12.

[11] T. Kubota, Nucl. Phys. B165 (1980) 277; L. Baulieu, E.G. Floratos, C. Kounnas, Phys. Lett. B89 (1979) 84, Nucl. Phys. B166 (1980) 321, Phys. Rev. D23 (1981) 2464; B. Humpert and W.L. van Neerven, (1980) unpublished.

[12] N.J.A. Sloane and S. Plouffe, The Encyclopedia of Integer Sequences, (Academic Press, San Diego, 1995). 


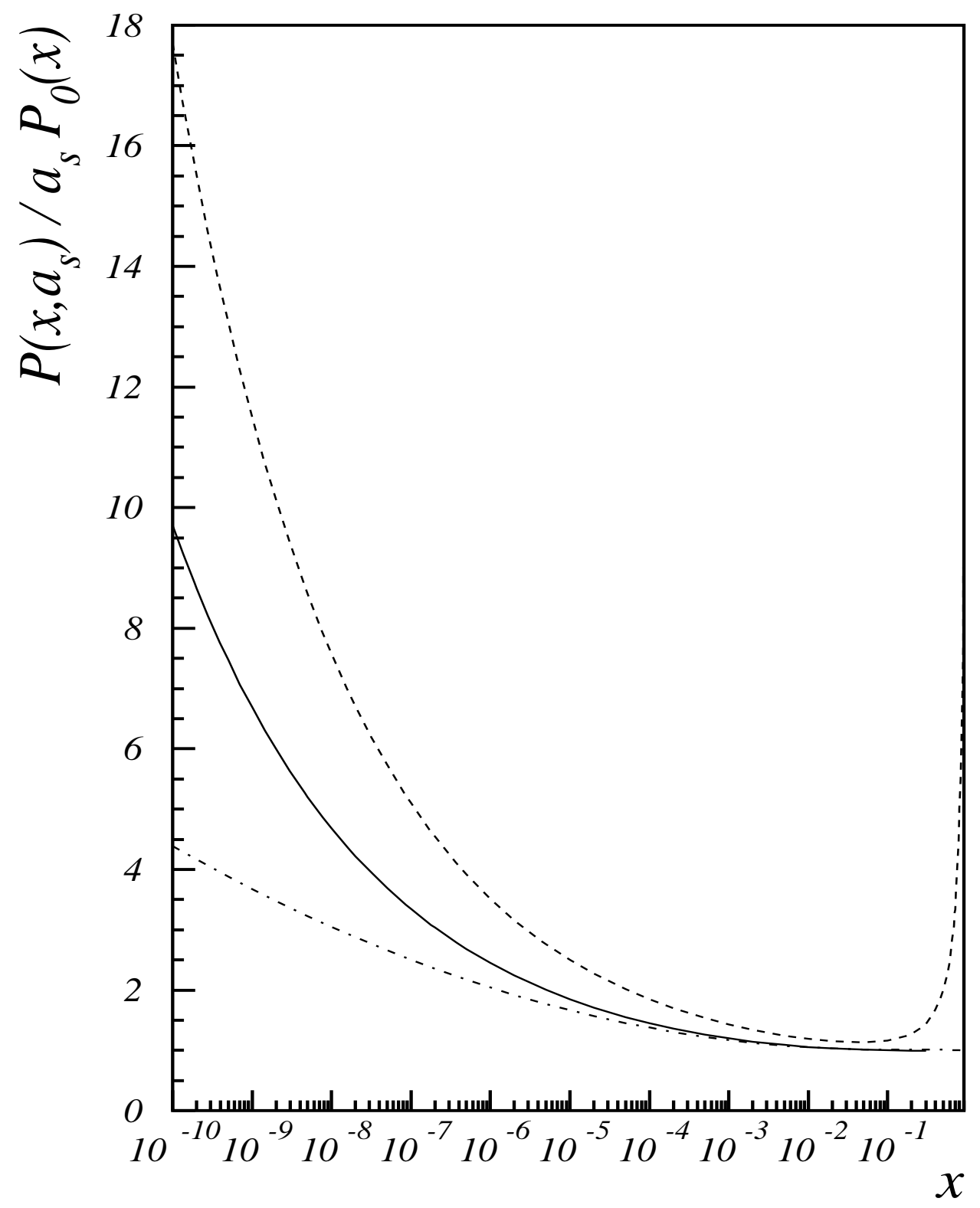

Figure 1: Fixed-order and resummed splitting functions $P\left(x, a_{s}\right)$ normalized to $a_{s} P_{S S}^{(0)}(x)$ for $\alpha_{s}=0.2$. Dash-dotted line : $P=a_{s} P_{L}^{(0)}+a_{s}^{2} P_{S S}^{(1)}$ Eqs. (7), (8). Solid line : $P=P_{L}$, Eq. (3). Dashed line: $P=P_{L, x \rightarrow 0}$, Eq. (11). 


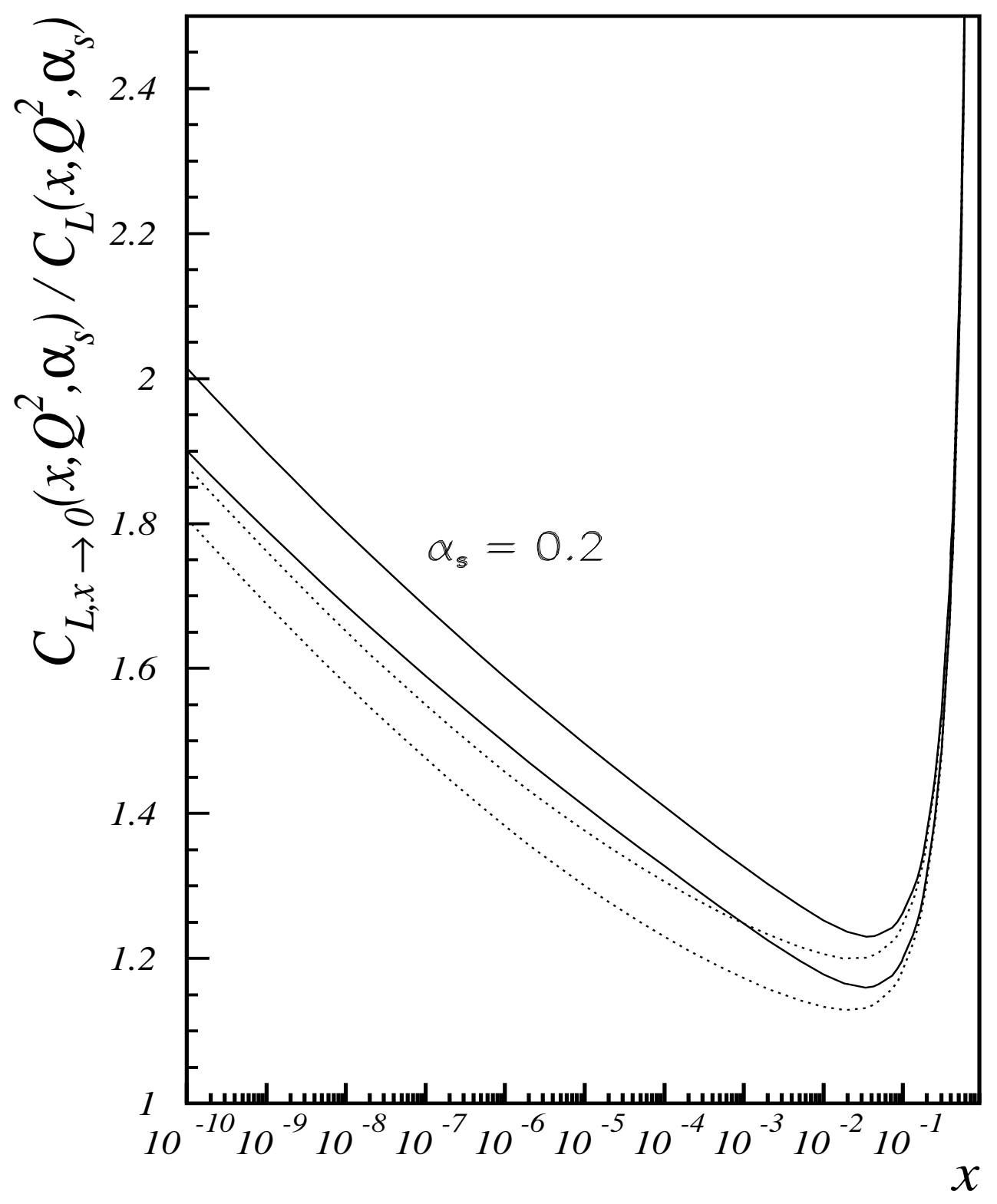

Figure 2: Ratio of the coefficient functions $C_{L, x \rightarrow 0}\left(x, Q^{2}, \alpha_{s}\right) / C_{L}\left(x, Q^{2}, \alpha_{s}\right)$, with $Q_{0}^{2}=4(\mathrm{GeV} / c)^{2}$ and $\alpha_{s}=0.2$. Solid lines : $C_{L}$ Eq. (3), $C_{L, x \rightarrow 0}$ Eq. (11). Dotted lines: To $C_{L}$ is added the contribution due to Eq. (10). The upper and lower lines correspond to $Q^{2}=10^{4}(\mathrm{GeV} / c)^{2}$ and $Q^{2}=10^{2}(\mathrm{GeV} / c)^{2}$ respectively. 